\title{
Interfacial rearrangements and strain evolution in the thin film growth of ZnPc on glass
}

\author{
Thomas L. Derrien $\odot^{*}$ \\ Diamond Light Source, Didcot, Oxfordshire OX11 ODE, United Kingdom \\ Andreas E. Lauritzen $\odot$ \\ Clarendon Laboratory, Department of Physics, University of Oxford, Oxfordshire, OX1 3PU, United Kingdom \\ Pascal Kaienburg $\odot$ \\ Clarendon Laboratory, Department of Physics, University of Oxford, Oxfordshire, OX1 3PU, United Kingdom \\ Ellis Hancox () \\ Department of Chemistry, University of Warwick, Gibbet Hill, Coventry CV4 7AL, United Kingdom \\ Chris Nicklin \\ Diamond Light Source, Didcot, Oxfordshire OX11 ODE, United Kingdom \\ Moritz Riede ${ }^{\dagger}$ \\ Clarendon Laboratory, Department of Physics, University of Oxford, Oxfordshire, OX1 3PU, United Kingdom
}

(Received 3 September 2021; accepted 15 February 2022; published 8 March 2022)

\begin{abstract}
We report on the characterization of the growth of vacuum-deposited zinc phthalocyanine ( $\mathrm{ZnPc}$ ) thin films on glass through a combination of in situ grazing incidence $\mathrm{x}$-ray scattering, $\mathrm{x}$-ray reflectivity, and atomic force microscopy. We found that the growth at room temperature proceeds via the formation of two structurally unique substrate-induced interfacial layers, followed by the growth of the $\gamma$-ZnPc polymorph thereafter (thickness $\approx 1.0$ $\mathrm{nm})$. As the growth of the bulk $\gamma$-ZnPc progresses, a substantial out-of-plane lattice strain $(\approx 15 \%$ relative to $\gamma$-ZnPc powder) is continually relaxed during the thin film growth. The rate of strain relaxation was slowed after a thickness of $\approx 13 \mathrm{~nm}$, corresponding to the transition from layer growth to island growth. The findings reveal the real-time microstructural evolution of $\mathrm{ZnPc}$ and highlight the importance of substrate-induced strain on thin film growth.
\end{abstract}

DOI: 10.1103/PhysRevMaterials.6.033401

\section{INTRODUCTION}

Organic electronics, based on vacuum-deposited small molecule organic semiconductors (OSCs) have been used to successfully build devices such as organic field-effect transistors (OFETs) [1,2], organic light emitting diodes (OLEDs) [3], and organic photovolataics (OPVs) [4-6]. While OLEDs have met commercial success due to their high efficiencies, sufficient lifetimes, and the scalability of vacuum deposition, the performance of OFETs and OPVs still lags behind those of inorganic devices. This is partially due to the complex mechanisms by which thin film OSCs develop, where elaborate

\footnotetext{
*Current address: Living Systems Institute, University of Exeter, Exeter EX4 4QD, United Kingdom; thomas.derrien@diamond.ac.uk

${ }^{\dagger}$ Corresponding author: moritz.riede@physics.ox.ac.uk

Published by the American Physical Society under the terms of the Creative Commons Attribution 4.0 International license. Further distribution of this work must maintain attribution to the author(s) and the published article's title, journal citation, and DOI.
}

microstructures occur across several length scales and can evolve as the thin film grows [7-11]. Device microstructure is critical for OPV and OFET performance and controlling the microstructure has even been found to improve OLED properties $[12,13]$. Of particular importance is the substrate on which these films are deposited, which can serve as a templating layer to influence the thin film morphology [14]. Other methods to control the morphology are chemical substitution [15], substrate temperature [16], and the application of light [17]. As the morphology of the active layer can have profound effects on device performance, it is critical to develop a thorough understanding of the parameters controlling the morphology of OSCs in vacuum-deposition [18,19].

Zinc phthalocyanine $(\mathrm{ZnPc})$ is an OSC which has been used extensively in OPV devices due to its inertness, chemical stability, and high absorption coefficient $\left(\varepsilon>10^{5} \mathrm{~cm}^{-1}\right.$ at $\approx$ $675 \mathrm{~nm}$ ) [20]. It has been shown to have several polymorphs [21] with $\gamma$ (or $\alpha$-II) and $\beta$ polymorphs often reported in device structures $[16,22,23]$. The crystalline structure of the photoactive layer as well as the crystalline orientation can have strong effects on solar cell performance [24] and this can be controlled by changing the substrate upon which the thin film is grown [25] or the temperature at which it is deposited 
[16]. In addition, this microstructure can vary throughout the film [16], in particular, near the substrate interface. This is of particular importance for OLEDs and OPVs as this interface is where charge injection and extraction occurs and the molecular orientation influences the interface energetics [26] as well as for OFET devices where charge transport is thought to be confined to the first few layers [27].

Here we report on the microstructural evolution of $\mathrm{ZnPc}$ thin films through the use of several characterization techniques. In situ grazing incidence x-ray scattering (GIXS) was used to probe the morphology of the growing film in realtime, revealing (1) the existence of two structurally unique interfacial layers and (2) strain relaxation throughout the thin film growth. Ex situ X-ray reflectivity (XRR) enabled the characterization of the layer morphology, confirming the existence of interfacial layers, and atomic force microscopy (AFM) provided direct imaging of the deposited layers.

\section{METHODS}

\section{A. Grazing incidence x-ray scattering}

In situ GIXS measurements were performed at the Diamond Light Source surface diffraction beamline (I07) [28] using an energy of $20 \mathrm{keV}$ in the purpose-built MINERVA sample chamber [29]. ZnPc evaporation in MINERVA was achieved with a low-temperature thermal evaporation source at a rate of $0.26 \pm 0.03 \AA / s$ as monitored by a water cooled quartz crystal microbalance (QCM), previously calibrated using ellipsometry. The evaporation was performed on Corning Eagle XG substrates (manufactured with a root mean square (RMS) roughness of $5 \AA$, low roughness confirmed by the lack of discernible diffuse scattering), which was cleaned in an ultrasonic bath for 10 min using a $2.5 \%$ Hellmanex (nonetching) solution, deionized water, acetone, and isopropanol, followed by a final UV-ozone cleaning. The cleaned substrate was mounted, and the chamber was pumped down to a pressure of $10^{-7}$ mbar prior to $\mathrm{ZnPc}$ evaporation. GIXS measurements were recorded every $10 \mathrm{~s}$ using an exposure time of $1 \mathrm{~s}$ at an incidence angle of $0.05^{\circ}$ (below the critical angle of the glass substrate $\alpha_{c} \approx 0.097^{\circ}$, and that of $\mathrm{ZnPc} \alpha_{c} \approx 0.082^{\circ}$ ). No beam damage was detected for thick films, as verified by comparing the $\mathrm{X}$-ray scattering images of the illuminated section with an off-beam section after the deposition. Images were collected using a Pilatus $2 \mathrm{M}$ detector at a sample to detector distance of $0.41 \mathrm{~m}$ that was calibrated using a silver behenate standard (AgBeh). Data reduction was performed in DAWN [30].

\section{B. X-ray reflectivity}

Ex situ XRR traces, performed at the Diamond Light Source surface diffraction beamline (I07), were recorded at 20 $\mathrm{keV}$ using a Pilatus $100 \mathrm{k}$ and monitoring the specular reflected beam at $2 \theta=0.085-2.15^{\circ}\left(q=0.15-0.38 \AA^{-1}\right)$. Background subtraction was performed by removing the scaled sum of the pixel values of the region selected outside the specular region. Fitting using the Paratt formalism was undertaken using GENX [31]. The samples were prepared in a separate evaporation chamber in the same manner as the in situ samples but the sample shutter was closed when the QCM readings reached the appropriate thicknesses $(1.8,3.5$, and $12.5 \mathrm{~nm})$.

\section{Atomic force microscopy}

AFM images were collected on the XRR samples using a Bruker Dimension Icon in tapping mode mounted with Bruker SCANASYST-AIR-HR tips. A scanning rate of $1 \mathrm{~Hz}$ was used and the images were analysed extract the roughness using GWYDDION [32].

\section{RESULTS}

To understand the growth of vacuum-deposited $\mathrm{ZnPc}$ on glass substrates, we utilized a combination of x-ray characterization and real space imaging. In situ GIXS was used to probe the layer growth as well as the crystalline structure of the growing film. Ex situ XRR enabled the characterization of the layer structure of $\mathrm{ZnPc}$, which was confirmed using AFM imaging.

The in situ GIXS experiments such as those employed here enable the characterization of intermediate microstructural states not normally accessible with ex situ techniques, such as transient polymorphs or interfacial phases not observable in thick films. Using this setup, we were able to monitor both the evolution of the crystalline structure and the growth mode of $\mathrm{ZnPc}$ as a function of film thickness during the thin film deposition. A surface plot of the out-of-plane scattering intensity at $q_{\|}=0$ of the first $30 \mathrm{~nm}$ of $\mathrm{ZnPc}$ is shown in Fig. 1(b). From the plot, two main features can be discerned: (1) the oscillation of the intensity in the low $q$ and (2) the increasing intensity of the Bragg peak at $q_{\|} \approx 0.49 \mathrm{~A}^{-1}$.

We attribute the oscillations in the low $\mathrm{q}$ to the layer-bylayer growth regime previously reported for $\mathrm{ZnPc}$ [33]. The oscillations, when recorded at the anti-Bragg point (where $q_{\perp}=0.25 \AA^{-1}$ and $\left.q_{\|}=0 \AA^{-1}\right)\left(q_{\perp}=\frac{1}{2} q_{\text {Bragg }}\right)$, occur with a period of two monolayers (MLs) [34]. The intensity at the anti-Bragg point is plotted in Fig. 2. Here it can be seen that, after the oscillations corresponding to the first two monolayers (red lines), the periodicity of the oscillations occur with a thickness of (1.1-1.4 nm) which is roughly the height of that of an edge on $\gamma$-ZnPc molecule $(\approx 1.3 \mathrm{~nm})$. This disparity in expected thickness for the first two monolayers indicates a structural variation for these interfacial layers. Such behavior has been previously reported in the groundbreaking works by Loi [35] and Heringdorf [36], as well as for similar molecules on glass, such as a fluorinated copper phthalocyanine $\left(\mathrm{F}_{16} \mathrm{CuPc}\right)[11]$, but, to our knowledge, never for $\mathrm{ZnPc}$. We hypothesize that this is due to a change in the stacking angle of $\mathrm{ZnPc}$ molecules implying a more face-on orientation than in subsequent layers. This implies a non-negligible interaction between $\mathrm{ZnPc}$ and glass, which is commonly thought of as an inert substrate.

To further probe the interlayer structure of the $\mathrm{ZnPc}$ films on glass, XRR was performed on ex situ samples of 1.8-, 4.3-, and 12.5-nm thickness (as measured by XRR). Fittings of the reflectivity revealed scattering length density (SLD) variations near the substrate which then reached the SLD of the bulk further from the interface. The low SLD interfacial layer mirrors that which was previously reported by De Oteyza et al. [11] 


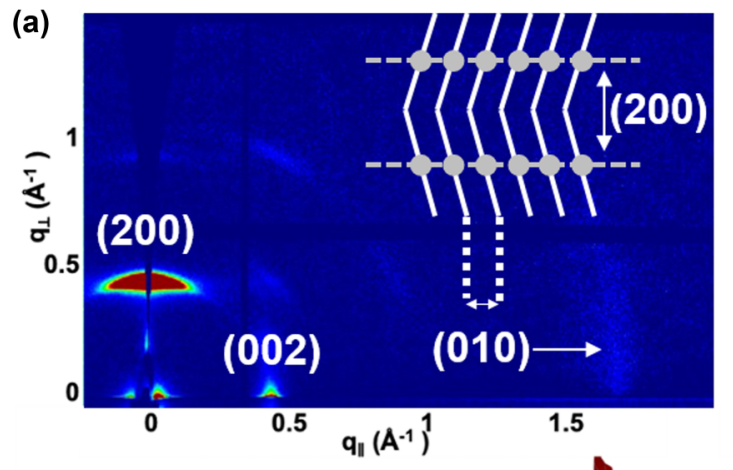

(b)

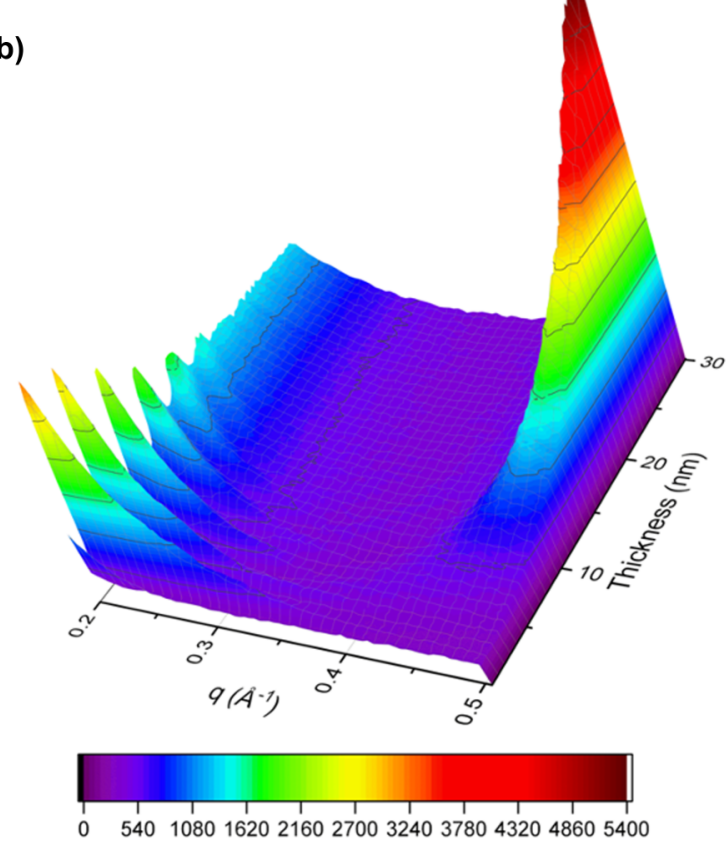

FIG. 1. (a) 2D GIXS image in reciprocal space of an 80-nm ZnPc film showing the Bragg peaks of interest and a schematic diagram of $\mathrm{ZnPc}$ molecular stacking (where the grey circles represent the $\mathrm{Zn}$ atom). (b) Surface plot of in situ grazing incidence x-ray scattering of $\mathrm{ZnPc}$ thin film on glass as a function of film thickness showing oscillations near the specular scatterings and the growing (200) peak. The plot was constructed from out-of-plane line cuts taken from $q_{\|}=0$.

for $\mathrm{F}_{16} \mathrm{CuPc}$ films, implying the existence of interfacial layers with lower molecular packing density than the subsequent $\mathrm{ZnPc}$ layers. The thickness of the low SLD layer closely matches that of the intensity oscillations measured by in situ GIXS, confirming the presence of the interfacial layers. As the thickness of the $\mathrm{ZnPc}$ film increases, the SLD of the interfacial layer increases, without significant change in the layer thickness, suggesting additional $\mathrm{ZnPc}$ molecules packing into the underlying interfacial layers as the film grows. This is distinct from the interfacial flat-lying $\alpha$-sexithiophene [35] and pentacene [36] layers previously observed, where the inclusion of additional molecules changes the orientation, and thereby the thickness of the interfacial layers. The XRR fits also showed increasing roughness for the growing films (Table I), where the thinner 2.0- and 3.5-nm films had a roughness of $0.40 \pm 0.15$ and $0.48 \pm 0.3 \mathrm{~nm}$, respectively, and the thicker, $12.5-\mathrm{nm}$ film showed a roughness approximately twice that of the thinner films $(0.9 \pm 0.3 \mathrm{~nm})$.

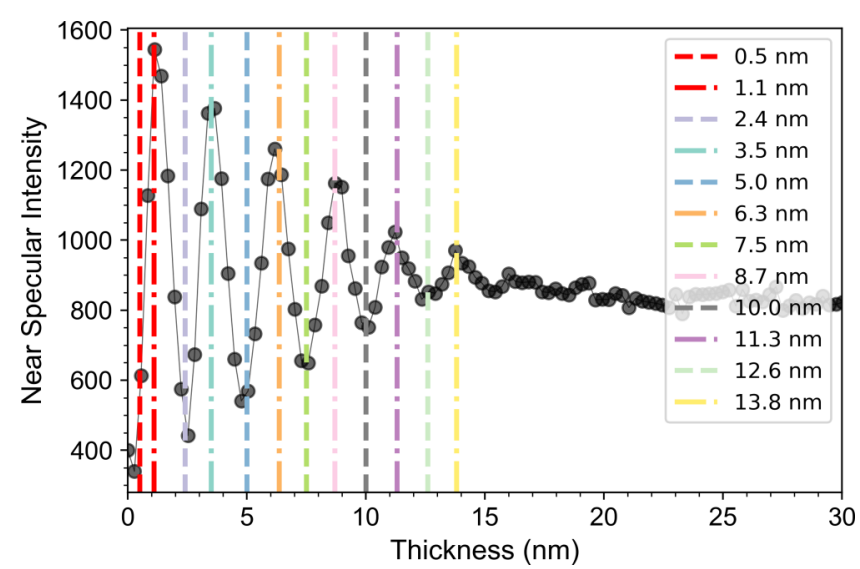

FIG. 2. Real-time oscillation of the diffuse scattering at the antiBragg point (where $q_{\perp}=0.25 \AA^{-1}$ and $q_{\|}=0 \AA^{-1}$ ) taken during ZnPc thin film growth. The dashed lines indicate the minima and maxima of the growth oscillations, corresponding to full $\mathrm{ZnPc}$ monoloayers.

The intensity variations in Fig. 2 as discussed above correspond to layer-by-layer growth of the growing $\mathrm{ZnPc}$ film. However, these oscillations dampened during the course of the thin film growth and had nearly disappeared by a thickness of $\approx 15 \mathrm{~nm}$. This is in line with the Stranksi-Krastanov (SK) growth mode previously reported [33] for $\mathrm{ZnPc}$, where SK growth transitions from layer-by-layer growth to island growth due to strain relief. This implied that the strain had reached a critical point at approximately 11-ML thickness, slightly larger than the 8 MLs reported by Kim et al. [33], though we begin to see major dampening occurring after $10 \mathrm{~nm}$ have been deposited (corresponding to $\approx 8 \mathrm{MLs}$ ). The dampening of the growth oscillations, due to roughening of the growing thin film, is consistent with the increased roughness [10] seen in the XRR calculation above for the 12.5-nm-thick sample. The evolution of the out of plane lattice strain could be tracked by monitoring the (200) peak position during the course of the $\mathrm{ZnPc}$ deposition. This was accomplished by taking line cuts from each individual 2D detector image and fitting the peak to a Lorentzian on a Gaussian background. The (200) Bragg peak was detectable after $\approx 4$ $\mathrm{nm}$ of $\mathrm{ZnPc}$ was deposited, enabling the calculation of the out-of-plane lattice strain relative to the bulk powder $d$ spacing $\left(d_{0}=12.9 \AA\right)$ [37] via Eq. (1).

$$
\epsilon_{h k l}=\left(\frac{d-d_{0}}{d_{0}}\right)_{h k l},
$$

where $\epsilon_{h k l}$ is the calculated lattice strain for an $h k l$ plane, $d$ is the calculated plane spacing, and $d_{0}$ is the bulk powder plane spacing.

TABLE I. Roughness as calculated from XRR (Fig. 3) and AFM (Fig. 6). XRR error was calculated from fits for XRR and from the standard deviation of the calculated RMS for AFM.

\begin{tabular}{lcc}
\hline \hline Sample thickness $(\mathrm{nm})$ & $\sigma_{\mathrm{XRR}}(\mathrm{nm})$ & $\sigma_{\mathrm{AFM}}(\mathrm{nm})$ \\
\hline 2.0 & $0.40 \pm 0.15$ & $0.32 \pm 0.7$ \\
3.5 & $0.48 \pm 0.3$ & $0.39 \pm 0.6$ \\
12.5 & $0.9 \pm 0.3$ & $0.81 \pm 0.5$ \\
40 & - & $2.42 \pm 0.4$ \\
\hline \hline
\end{tabular}



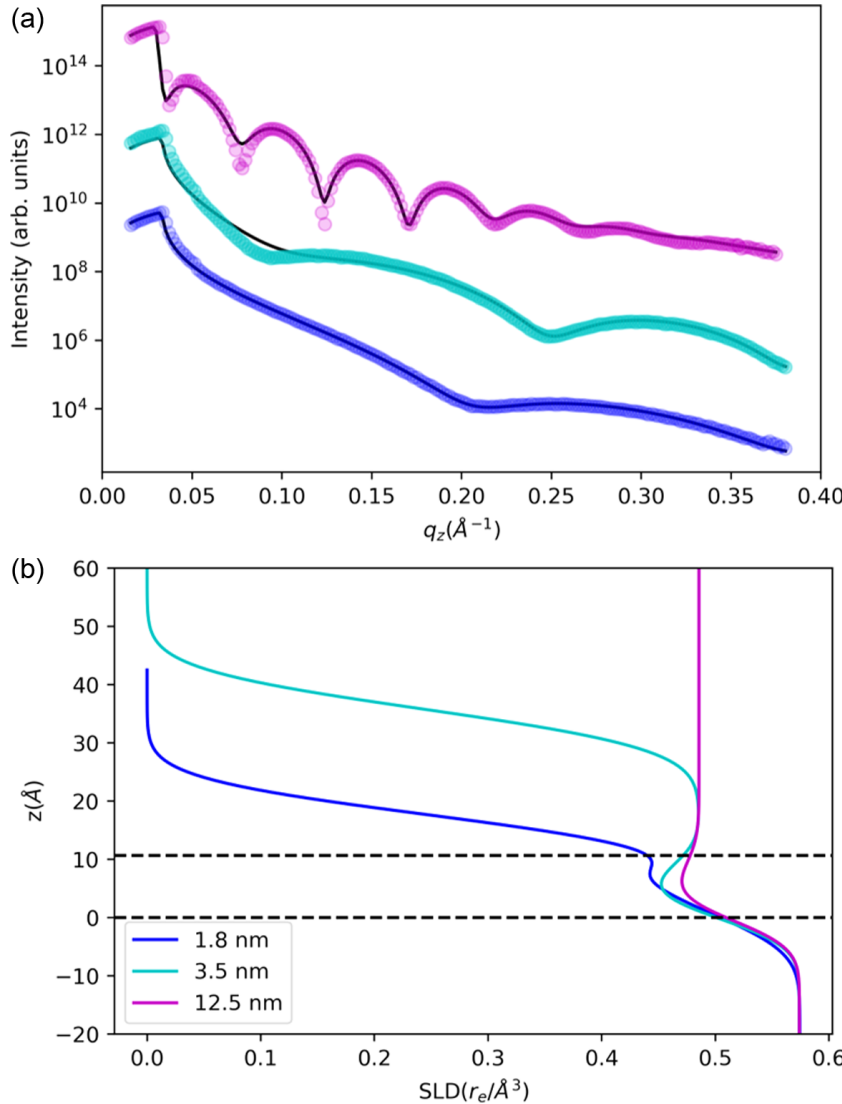

FIG. 3. (a) X-ray reflectivity measurements and fits of ZnPc films of 1.8-, 3.5-, 12.5-nm thickness. (b) Scattering length density profiles calculated from the $\mathrm{x}$-ray reflectivity fits with dashed lines showing the thickness of the interfacial layer.

The peak position and 200 lattice strain are plotted in Fig. 4. Here it can be seen that the 200 peak position increased quickly in the early stages of the ZnPc deposition before the rate of change slowed at around a thickness of 10-15 nm. This could be captured by a dual-exponential model, as fitting the curve to a single exponential could not accurately capture the behavior in this transition region. This suggests that various growth modes are present at different stages of the film growth, as present in the aforementioned SK growth. Indeed, the transition region between the two exponentials $(\approx 10-15 \mathrm{~nm})$ occurs when the oscillations in Fig. 2 are almost completely dampened. Interestingly, this region also corresponds to the thickness where the lattice strain, relative to the bulk powder, reaches zero [Fig. 4(b)], and we expect they are linked. After this point, the strain (with respect to the bulk powder spacing) continues to increase albeit at a much slower rate. To merge the two exponential growth regions and extract quantitative information about the transition region, an error function was applied to fit the transition region. The exponential from the early time points $\left(q_{1}\right)$ and the subsequent exponential $\left(q_{2}\right)$ were fit using Eqs. (2) and (3), respectively,

$$
\begin{aligned}
& q_{1}=q_{1_{\text {max }}}\left(1-e^{-k_{1}\left(t-c_{1}\right)}\right), \\
& q_{2}=q_{2_{\text {max }}}\left(1-e^{-k_{2}\left(t-c_{2}\right)}\right),
\end{aligned}
$$
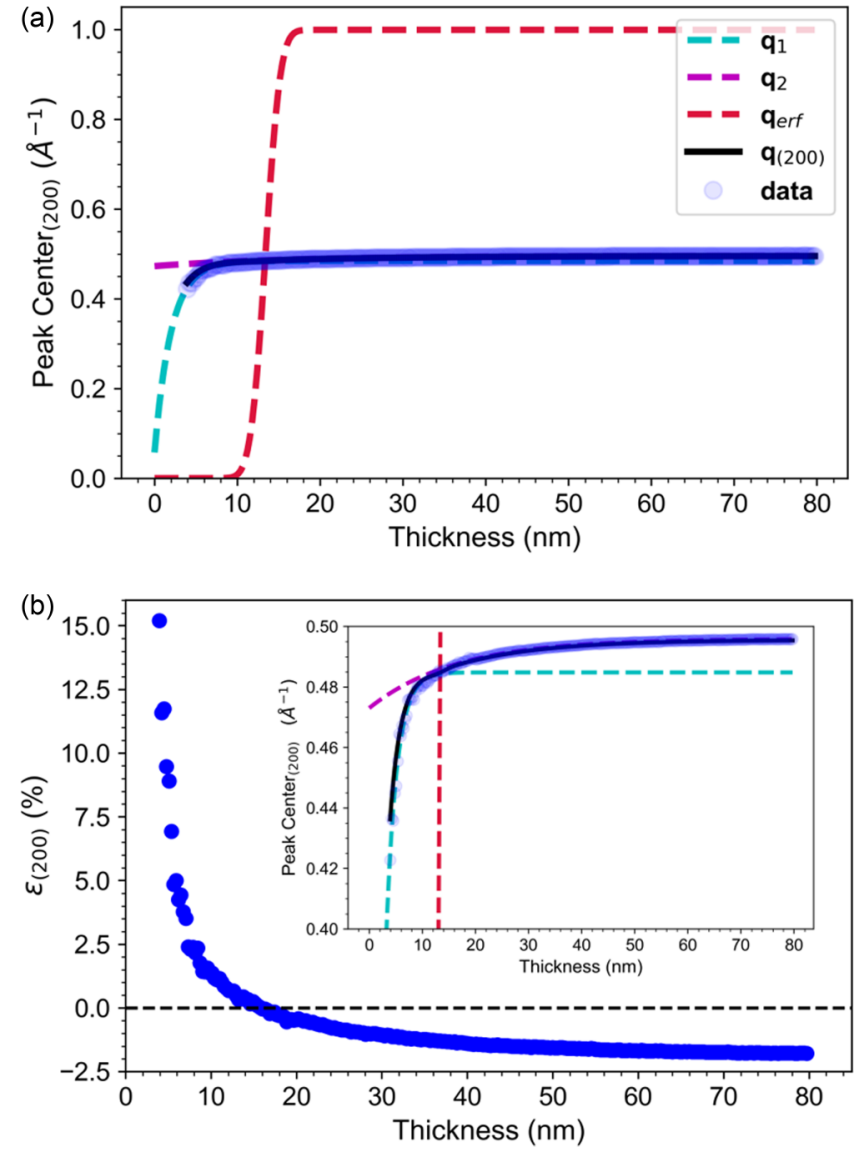

FIG. 4. (a) Evolution of the ZnPc (200) peak position during vacuum deposition fit to a dual exponential model. (b) The out-of-plane lattice strain (relative to bulk powder) progression during thin film growth. Inset: Dual-exponential fit from (a) scaled to show details of individual exponential fit components $\left(q_{1}\right.$ and $\left.q_{2}\right)$ and the modified error function, $q_{\text {erf }}$, used to merge the two compenents.

where $\left(q_{1_{\max }}\right)$ and $\left(q_{2_{\max }}\right)$ are coefficients describing the scale of the exponential, $t$ is the film thickness in $\mathrm{nm}, k_{1}$ and $k_{2}$ are exponential decay constants, and $c_{1}$ and $c_{2}$ are offsets.

To fit the evolution of the (200) peak across the entire thickness range, the sum of $q_{1}$ and $q_{2}, q_{(200)}$ [Eq. (4)] is formed,

$$
q_{(200)}=q_{1}\left(1-q_{\mathrm{erf}}\right)+q_{\mathrm{erf}} q_{2},
$$

where a smeared out step function in the form of an error function,

$$
q_{\text {erf }}=\frac{1}{2}\left[K_{\text {erf }} \operatorname{erf}\left(w\left(t-c_{\text {erf }}\right)\right)+K_{\text {erf }}\right],
$$

is applied as a weighting factor to smoothen the transition between the two growth regimes, where $K_{\text {erf }}$ is a scaling coefficient of the error function, $w$ is the width of the error function, and $c_{\text {erf }}$, is an offset.

The resulting fit can be seen in Fig. 4(a), where the exponential $\left(q_{1}, q_{2}\right)$ and the modified error function $\left(q_{\text {erf }}\right)$ components are also plotted to form the final growth function, $q_{(200)}$ [the inset in Fig. 4(b) is scaled to show the details of the fitting components]. A strong fit to the experimental data was achieved using this modular growth model. Upon inspection 


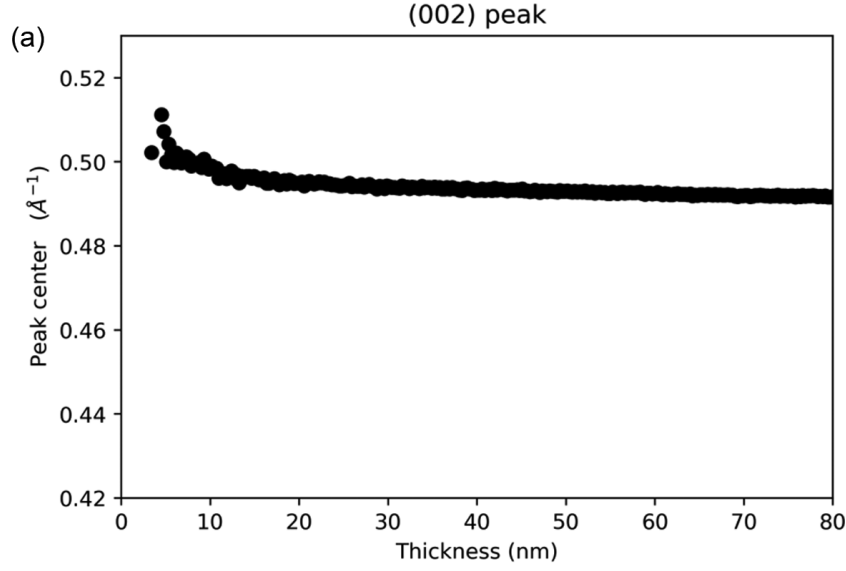

(b)

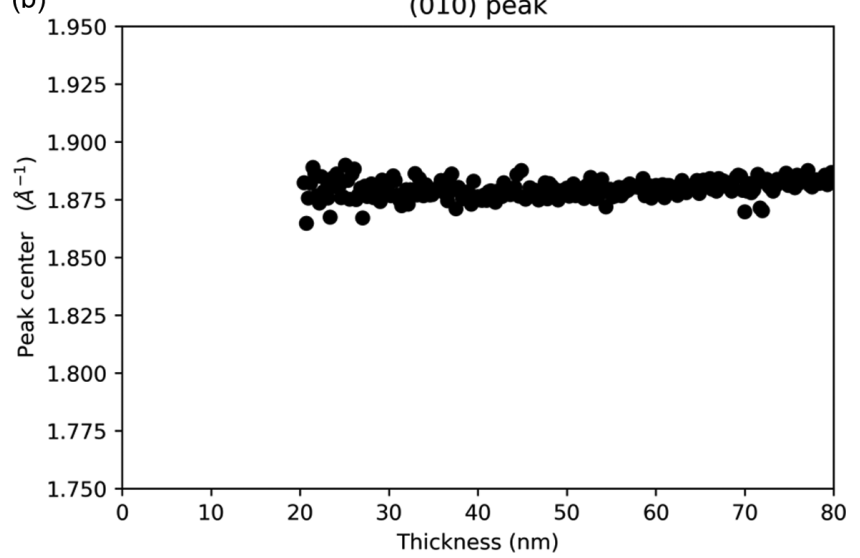

FIG. 5. (a) Plots of 002 and (b) 010 (pi-pi stacking) peak positions (in plane).

of the resultant fit parameters, a steep slope in the error function which merges the two growth modes can be seen. This steep slope of the error function implies that the transition between the early stage layer-by-layer growth mode and the late stage island growth mode occurred abruptly. Quantitative information about the width of this transition region was estimated using the FWHM of the derivative of $q_{\text {erf }}$ [38]. Indeed, the estimated 3.5-nm width denotes a rapid transition. This transition, centered around $13.4 \mathrm{~nm}$, is in agreement with the region in which the growth oscillations seen in Fig. 2 are almost completely dampened $(\approx 15 \mathrm{~nm})$.

The in-plane lattice strain could also be inferred using the same method described above by monitoring the position of the (002) Bragg peak (Fig. 1). In contrast to the out-of-plane (200) peak which showed substantial increase in the $q$ value, the position of the (002) peak (once resolved for thickness $\gtrsim 3 \mathrm{~nm}$ ) initially shifted to slightly lower values before remaining constant throughout the bulk thin film deposition [Fig. 5(a)]. Similarly, the (010) peak, which corresponds to the pi-pi stacking between neighboring $\mathrm{ZnPc}$ molecules, showed no peak shift throughout the deposition (though the peaks were not resolvable for thicknesses $\lesssim 20 \mathrm{~nm}$, corresponding to the region of slower change in the (200) peak). The contrasting behavior of the (200) and (002) peaks shows that as the interplanar spacing is compressed throughout most of the course of the deposition [(200) peak shift], this is accompanied by only a slight lengthening in the in-plane intermolecular spacing occurring only at the early stages of $\mathrm{ZnPc}$ deposition [(002) peak shift]. The range of these shifts, however, differs in magnitude; while the (200) peak shift corresponds to a compression from $14.35 \AA$ to $12.67 \AA$, the (002) peak corresponds to a lengthening from $12.29 \AA$ to 12.77 $\AA$. As the transition from layer-by-layer to island growth is mediated by strain relief in SK growth, this implies that for $\mathrm{ZnPc}$ thin films on glass, this is controlled primarily by the out-of-plane strain.

The X-ray findings were corroborated in real space with AFM imaging. Four thicknesses were imaged (2.0-, 3.5-, 12.5-, and 40.0-nm-thick films). The images show the progression from smooth films $(2.0,3.5 \mathrm{~nm})$ to textured films showing islands $(12.5 \mathrm{~nm}, 40 \mathrm{~nm})$. The image analysis revealed the roughness of the films increased with thickness (Table I). This can clearly be seen in the profiles below the image. Interestingly, the 12.5-nm image, a thickness near the transition from LbL to island growth, shows the presence of small islands, which became larger and increased in roughness for the 40.0-nm-thick film. The roughness measured by XRR was consistent with but slightly larger than that measured using AFM, which we attribute to the smaller sampling area of the sample than with XRR $\left(0.25 \mu \mathrm{m}^{2}\right.$ for a $500 \times 500 \mathrm{~nm}$ AFM versus an estimated $9 \times 10^{4} \mu \mathrm{m}^{2}$ for a $300 \times 300 \mu \mathrm{m}$ beam at the highest angles probed). The AFM roughness data
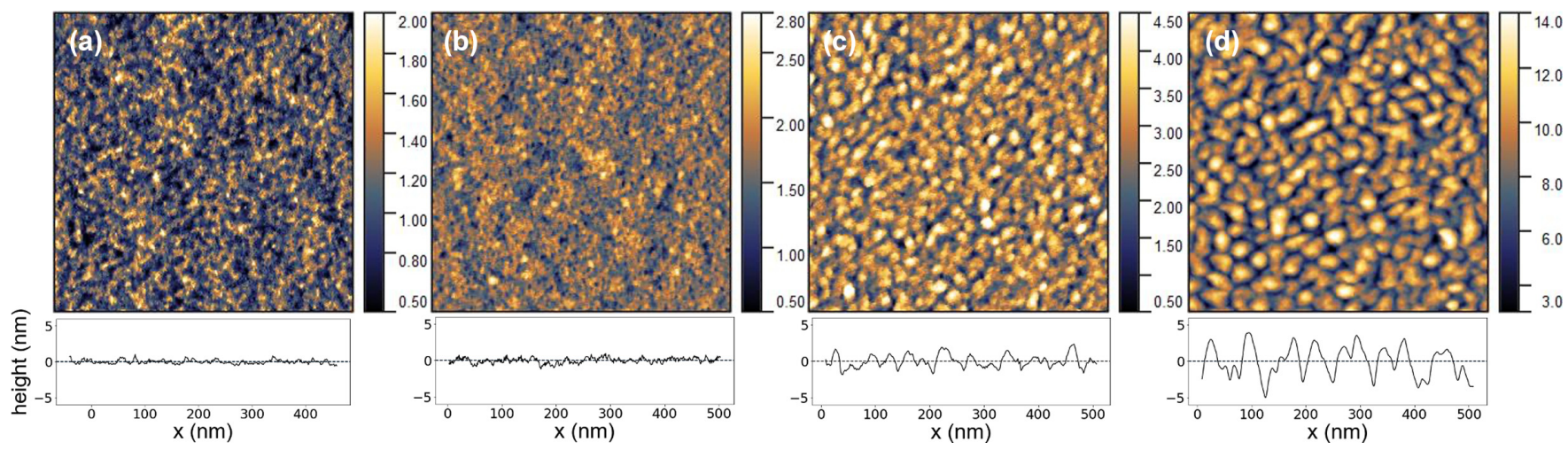

FIG. 6. AFM and corresponding line profiles (taken from $y=250 \mathrm{~nm}$ ) of $\mathrm{ZnPc}$ thin films of (a) 2, (b) 3.5, (c) 12.5, and (d) 40-nm thicknesses. Each image is $500 \times 500 \mathrm{~nm}$. 
were used to calculate the growth exponent, $\beta$, according to the scaling theory of growth-induced roughness [39,40]. A value of $\beta=0.67 \pm .03$ was found [38], corresponding to thin film growth with a rapid roughening process (i.e., $\beta \geqslant 0.5$ ). Though this has not been reported for $\mathrm{ZnPc}$, this is in line with previous finding on OSCs on $\mathrm{SiO}_{2}$ substrates [39,41], including the fluorinated phthalocyanine $\mathrm{F}_{16} \mathrm{CuPc}$ [7].

\section{CONCLUSION}

Our findings on a model system of a polycrystalline small molecule film evaporated in vacuum highlight the importance of independently probing the interface microstructure as it cannot be inferred from the bulk microstructure. In summary, our results show the ambient temperature growth of vacuumdeposited $\mathrm{ZnPc}$ on glass proceeds via a SK growth mode, with two structurally unique layers occurring at the substrate interface. Furthermore, the transition from layer-by-layer to island growth occurs after $\approx 11$ MLs have been deposited. This transition occurs at a film thickness of $\approx 13 \mathrm{~nm}$, after which the strain changes as a function of the film thickness for the growing films is considerably reduced. The growth kinetics were fit with a dual-exponential model and the width of this transition region was estimated to be $\approx 3.5 \mathrm{~nm}$. The results show the importance of the substrate-induced strain on the morphology of OSCs. The applied ex situ and in situ methods (GIXS, XRR, AFM) are well suited to study relevant microstructure at the interface and can be extended from our model system to other materials, including to some degree, amorphous materials. Additional in situ studies on OSC blends and on device-relevant substrates with upgraded chamber capabilities are expected to advance the development of key structure-property relationships to improve OSC device performance.

Elucidating the parameters which control OSC microstructure is key to advancing the field, as microstructural differ- ences are directly related to (opto)electronic properties and OSC device performance, e.g., the observed change of strain and molecular orientation is likely to cause, among other things, a shift in energy level alignment [42] with any underlying contact material which then affects charge injection and extraction in OLEDs and OPV. For OFETs where the electronic function is confined to a thin interfacial channel, the interfacial layer is even more important. The existence of a substrate-induced interfacial layer as well as a gradual transition to bulk morphology and bulk properties over $\approx 10 \mathrm{~nm}$ should be accounted for in electric device modeling as for certain aspects mentioned above, interface microstructure is crucial for understanding OSC device behavior.

\section{ACKNOWLEDGMENTS}

T.L.D., P.K., C.N., and M.R. acknowledge funding for this paper from the UKRI-GCRF grant Synchrotron Techniques for African Research and Technology (START) No. ST/R002754/1. A.E.L. thanks EPSRC for funding through the Doctoral Training Partnership (No. EP/N509711/1) as well as STFC and the ISIS Neutron and Muon facility and project (No. 1948713). Access to Diamond beamtime at I07 was provided under Experiment No. SI20426-1. The authors thank A. Warne and J. Rawle, H. Hussain, and F. Carla (Diamond Light Source) for their assistance with beamline instrumentation. We are grateful to J. Naylor, D. Wicks, and A. Dorman of K. J. Lesker Ltd. for generously lending deposition control and evaporation sources along with technical support for MINERVA which was the result of an STFC CLASP project (No. ST/L006294/1).

T.L.D. prepared the original draft with all authors contributing the reviewing and editing process. T.L.D., A.E.L., P.K., and E.H. performed the experiments and T.L.D. analyzed the data. The project was conceived by T.L.D., A.E.L., C.N., and M.R., with C.N. and M.R providing supervision.
[1] P. R. Warren, J. F. M. Hardigree, A. E. Lauritzen, J. Nelson, and M. Riede, Tuning the ambipolar behaviour of organic field effect transistors via band engineering, AIP Adv. 9, 035202 (2019).

[2] L. Torsi, M. Magliulo, K. Manoli, and G. Palazzo, Organic field-effect transistor sensors: A tutorial review, Chem. Soc. Rev. 42, 8612 (2013).

[3] D. Di, A. S. Romanov, L. Yang, J. M. Richter, J. P. H. Rivett, S. Jones, T. H. Thomas, M. Abdi Jalebi, R. H. Friend, M. Linnolahti, M. Bochmann, and D. Credgington, High-performance light-emitting diodes based on carbenemetal-amides, Science 356, 159 (2017).

[4] I. Ramirez, M. Causa', Y. Zhong, N. Banerji, and M. Riede, Key tradeoffs limiting the performance of organic photovoltaics, Adv. Energy Mater. 8, 1703551 (2018).

[5] O. Inganäs, Organic photovoltaics over three decades, Adv. Mater. 30, 1800388 (2018).

[6] G. Heliatek, www.heliatek.com/en/about.

[7] J. Yang, S. Yim, and T. S. Jones, Molecular-orientationinduced rapid roughening and morphology transition in organic semiconductor thin-film growth, Sci. Rep. 5, 9441 (2015).
[8] T. L. Derrien, A. E. Lauritzen, P. Kaienburg, J. F. M. Hardigree, C. Nicklin, and M. Riede, In situ observations of the growth mode of vacuum-deposited $\alpha$-sexithiophene, J. Phys. Chem. C 124, 11863 (2020).

[9] C. Lorch, R. Banerjee, C. Frank, J. Dieterle, A. Hinderhofer, A. Gerlach, and F. Schreiber, Growth of competing crystal phases of $\alpha$-sexithiophene studied by real-time in situ x-ray scattering, J. Phys. Chem. C 119, 819 (2015).

[10] S. Kowarik, A. Gerlach, M. W. A. Skoda, S. Sellner, and F. Schreiber, Real-time studies of thin film growth: Measurement and analysis of $\mathrm{x}$-ray growth oscillations beyond the anti-Bragg point, Eur. Phys. J.: Spec. Top. 167, 11 (2009).

[11] D. G. De Oteyza, E. Barrena, S. Sellner, J. O. Ossó, and H. Dosch, Structural rearrangements during the initial growth stages of organic thin films of F16CuPc on SiO2, J. Phys. Chem. B 110, 16618 (2006).

[12] J. Cui, Q. Huang, J. Veinot, H. Yan, and T. Marks, Interfacial microstructure function in organic light-emitting diodes: Assembled tetraaryldiamine and copper phthalocyanine interlayers, Adv. Mater. 14, 565 (2002).

[13] V. N. Hamanaka, E. Salsberg, F. J. Fonseca, and H. Aziz, Investigating the influence of the solution-processing method on the 
morphological properties of organic semiconductor films and their impact on OLED performance and lifetime, Org. Electron. 78, 105509 (2020).

[14] T. Wang, T. R. Kafle, B. Kattel, Q. Liu, J. Wu, and W.-L. Chan, Growing ultra-flat organic films on graphene with a face-on stacking via moderate molecule-substrate interaction, Sci. Rep. 6, 28895 (2016)

[15] R. Fitzner, E. Reinold, A. Mishra, E. Mena-Osteritz, H. Ziehlke, C. Körner, K. Leo, M. Riede, M. Weil, O. Tsaryova, A. Weiß, C. Uhrich, M. Pfeiffer, and P. Bäuerle, Dicyanovinyl-substituted oligothiophenes: Structure-property relationships and application in vacuum-processed small molecule organic solar cells, Adv. Funct. Mater. 21, 897 (2011).

[16] C. Schünemann, C. Elschner, A. Levin, M. Levichkova, K. Leo, and M. Riede, Zinc phthalocyanine-influence of substrate temperature, film thickness, and kind of substrate on the morphology, Thin Solid Films 519, 3939 (2011).

[17] L. Pithan, C. Cocchi, H. Zschiesche, C. Weber, A. Zykov, S. Bommel, S. J. Leake, P. Schäfer, C. Draxl, and S. Kowarik, Light controls polymorphism in thin films of sexithiophene, Cryst. Growth Des. 15, 1319 (2015).

[18] Z. Zhang and Y. Lin, Organic semiconductors for vacuumdeposited planar heterojunction solar cells, ACS Omega 5, 24994 (2020).

[19] J. Locklin, M. E. Roberts, S. C. B. Mannsfeld, and Z. Bao, Optimizing the thin film morphology of organic field-effect transistors: The influence of molecular structure and vacuum deposition parameters on device performance, J. Macromol. Sci., Part C 46, 79 (2006).

[20] G. Chamberlain, Organic solar cells: A review, Sol. Cells 8, 47 (1983).

[21] T. Guo, T. Zou, P. Shi, Y. Song, M. Wu, F. Xiao, J. Zhang, W. $\mathrm{Wu}$, and $\mathrm{H}$. Wang, A new polymorph of zinc-phthalocyanine and its optical properties, J. Cryst. Growth 546, 125760 (2020).

[22] M. Kato, M. Nakaya, Y. Matoba, S. Watanabe, K. Okamoto, J.-P. Bucher, and J. Onoe, Morphological and optical properties of $\alpha$ - and $\beta$-phase zinc ( $\|$ ) phthalocyanine thin films for application to organic photovoltaic cells, J. Chem. Phys. 153, 144704 (2020).

[23] G. O. Ngongang Ndjawa, K. R. Graham, R. Li, S. M. Conron, P. Erwin, K. W. Chou, G. F. Burkhard, K. Zhao, E. T. Hoke, M. E. Thompson, M. D. McGehee, and A. Amassian, Impact of molecular orientation and spontaneous interfacial mixing on the performance of organic solar cells, Chem. Mater. 27, 5597 (2015).

[24] W. Zeng, K. S. Yong, Z. M. Kam, F. Zhu, and Y. Li, Effect of blend layer morphology on performance of ZnPc:c60-based photovoltaic cells, Appl. Phys. Lett. 97, 133304 (2010).

[25] C. Schünemann, D. Wynands, K.-J. Eichhorn, M. Stamm, K. Leo, and M. Riede, Evaluation and control of the orientation of small molecules for strongly absorbing organic thin films, J. Phys. Chem. C 117, 11600 (2013).

[26] E. J. G. Santos and W. L. Wang, Ultrafast charge-transfer in organic photovoltaic interfaces: Geometrical and functionalization effects, Nanoscale 8, 15902 (2016).
[27] D. Ji, L. Li, H. Fuchs, and W. Hu, Engineering the interfacial materials of organic field-effect transistors for efficient charge transport, Accounts Mater. Res. 2, 159 (2021).

[28] C. Nicklin, T. Arnold, J. Rawle, and A. Warne, Diamond beamline I07: A beamline for surface and interface diffraction, $\mathrm{J}$ Synchrotron Radiat. 23, 1245 (2016).

[29] C. Nicklin, J. Martinez-Hardigree, A. Warne, S. Green, M. Burt, J. Naylor, A. Dorman, D. Wicks, S. Din, and M. Riede, Minerva: A facility to study microstructure and interface evolution in realtime under vacuum, Rev. Sci. Instrum. 88, 103901 (2017).

[30] J. Filik, A. W. Ashton, P. C. Y. Chang, P. A. Chater, S. J. Day, M. Drakopoulos, M. W. Gerring, M. L. Hart, O. V. Magdysyuk, S. Michalik, A. Smith, C. C. Tang, N. J. Terrill, M. T. Wharmby, and $\mathrm{H}$. Wilhelm, Processing two-dimensional X-ray diffraction and small-angle scattering data in DAWN2, J. Appl. Crystallogr. 50, 959 (2017).

[31] M. Björck and G. Andersson, GenX: An extensible X-ray reflectivity refinement program utilizing differential evolution, J. Appl. Crystallogr. 40, 1174 (2007).

[32] D. Nečas and P. Klapetek, Gwyddion: An open-source software for SPM data analysis, Central Eur. J. Phys. 10, 181 (2012).

[33] H. J. Kim, J. W. Kim, H. H. Lee, B. Lee, and J.-j. Kim, Initial Growth Mode, Nanostructure, and molecular stacking of a ZnPc:C60 bulk heterojunction, Adv. Funct. Mater. 22, 4244 (2012).

[34] S. Kowarik, Thin film growth studies using time-resolved X-ray scattering, J. Phys.: Condens. Matter 29, 043003 (2016).

[35] M. A. Loi, E. da Como, F. Dinelli, M. Murgia, R. Zamboni, F. Biscarini, and M. Muccini, Supramolecular organization in ultra-thin films of $\alpha$-sexithiophene on silicon dioxide, Nat. Mater. 4, 81 (2005).

[36] F.-J. Meyer zu Heringdorf, M. C. Reuter, and R. M. Tromp, Growth dynamics of pentacene thin films, Nature (London) 412, 517 (2001)

[37] S. Senthilarasu, Y. B. Hahn, and S.-H. Lee, Structural analysis of zinc phthalocyanine $(\mathrm{ZnPc})$ thin films: X-ray diffraction study, J. Appl. Phys. 102, 043512 (2007).

[38] See Supplemental Material at https://link.aps.org/supplemental/ 10.1103/PhysRevMaterials.6.033401 for the fit of the derivative of $q_{\text {erf }}$, the growth exponent, and a time-lapse of the scattering images.

[39] A. C. Durr, F. Schreiber, K. A. Ritley, V. Kruppa, J. Krug, H. Dosch, and B. Struth, Rapid Roughening in Thin Film Growth of an Organic Semiconductor (Diindenoperylene), Phys. Rev. Lett. 90, 016104 (2003).

[40] J. Krug, Origins of scale invariance in growth processes, Adv. Phys. 46, 139 (1997).

[41] X. Zhang, E. Barrena, D. de Oteyza, and H. Dosch, Transition from layer-by-layer to rapid roughening in the growth of DIP on $\mathrm{SiO} 2$, Surf. Sci. 601, 2420 (2007).

[42] Y. Wu, A. R. Chew, G. A. Rojas, G. Sini, G. Haugstad, A. Belianinov, S. V. Kalinin, H. Li, C. Risko, J.-L. Brédas, A. Salleo, and C. D. Frisbie, Strain effects on the work function of an organic semiconductor, Nat. Commun. 7, 10270 (2016). 